\title{
Stationarity of electron distribution in ground-state molecular systems
}

\author{
Dariusz Szczepanik • Janusz Mrozek
}

Received: 10 December 2012 / Accepted: 15 February 2013 / Published online: 27 February 2013

(C) The Author(s) 2013. This article is published with open access at Springerlink.com

\begin{abstract}
Stationarity of electron probability distribution within the resolution of atomic orbitals is considered involving some concepts from Orbital Communication Theory and the theory of Markov Processes. A new method of evaluating electron conditional probabilities based on natural orbitals is proposed and briefly discussed.
\end{abstract}

Keywords Stationary distribution - Markov chain - Density matrix ·

Conditional probability $\cdot$ Natural orbital

\section{Introduction}

In the last half-century the electron population analyses (EPA) turned out to be very useful and commonly used tools in probing the electronic structure and chemical reactivity of molecules. In brief, EPA-procedures give rise to partition the electron density of the whole molecule between atoms, chemical bonds, molecular fragments, etc. Such partition can be performed within either physical or Hilbert space of molecular orbitals (MO) and there is a multitude of diverse EPA-procedures among which the most commonly used are those proposed by: Mulliken [1,2], Löwdin [3,4], Weinhold [5], Bader [6], Hirshfeld [7] and Merz-Kollman [8].

Although this is a field of research that seems to be regarded by some scientists as exhausted, in this short paper we would like to address some of the general issues respecting stationarity and uniqueness of electron probability distributions within the framework of MO theory and resolution of atomic orbitals (AO). Our considerations will be confined only to closed-shell ground states at two levels of theory,

D. Szczepanik $(\varangle) \cdot$ J. Mrozek

Department of Computational Methods in Chemistry, Faculty of Chemistry,

Jagiellonian University, R. Ingardena St. 3, 30-060 Cracow, Poland

e-mail: szczepad@chemia.uj.edu.pl 
Hartree-Fock (HF) and configuration interaction (CI) [9]. The main purpose of this article is to briefly introduce a new method of determining stationary electron probability distributions, based on natural orbitals (NO) and involving some concepts from the Orbital Communication Theory (OCT) of the chemical bond [10-12] and the theory of Markov Processes [13].

\section{Stationarity from idempotency}

For simplicity and transparency of definitions we assume without loss of generality the wavefunctions to be real. First, let us consider the one-determinant wavefunction of the ground-state molecular system, $\left|\Psi_{H F}\right\rangle$, with $N$ electrons doubly occupying the $n^{o}$ lowest of MOs, $\left|\Phi^{o}\right\rangle$, from the Hilbert space of the all $n$ orthonormal (spinless) molecular orbitals,

$$
|\boldsymbol{\Phi}\rangle=\left\{\left|\Phi_{1}^{o}\right\rangle, \ldots,\left|\Phi_{n^{o}}^{o}\right\rangle,\left|\Phi_{n^{o}+1}^{v}\right\rangle, \ldots,\left|\Phi_{n}^{v}\right\rangle\right\}
$$

generated as the linear combinations of basis functions $|\chi\rangle$, representing orthogonal atomic orbitals,

$$
|\boldsymbol{\Phi}\rangle=|\chi\rangle \mathbf{C}
$$

where square matrix $\mathbf{C}$ groups the relevant LCAO MO expansion coefficients,

$$
\mathbf{C}=\left(\mathbf{C}^{o} \mid \mathbf{C}^{v}\right)=\left\{C_{\mu, i}=\left\langle\chi_{\mu} \mid \Phi_{i}\right\rangle\right\}
$$

Obviously, $n=n^{o}+n^{v}$, and superscripts $o$ and $v$ refer to occupied and virtual MOsubspaces, respectively.

A set of such MOs uniquely defines the system one-electron density,

$$
\rho(\mathbf{r})=2 \sum_{i=1}^{n^{o}}|\boldsymbol{\Phi}(\mathbf{r})|^{2}=\chi(\mathbf{r}) \boldsymbol{\gamma} \chi^{\dagger}(\mathbf{r}),
$$

where $\gamma$ is the so called charge-and-bond-order (CBO) matrix,

$$
\gamma=2 \mathbf{C}^{o} \mathbf{C}^{\circ \dagger}, \quad \gamma_{\mu, \nu}=2 \sum_{i=1}^{n^{o}} C_{\mu, i} C_{v, i},
$$

satisfying the following idempotency relation appropriate for the closed-shell systems (duodempotency relation):

$$
\boldsymbol{\gamma}^{2}=2 \boldsymbol{\gamma}, \quad \text { or } \quad \sum_{\nu} \gamma_{\mu, \nu}^{2}=2 \gamma_{\mu, \mu}
$$


Diagonal elements of symmetric matrix CBO, $\left\{\gamma_{\mu, \mu}\right\}$, can be regarded as effective electron occupations of AOs, $N_{\mu}$, determining the corresponding electron probability in $\mathrm{AO}$ resolution:

$$
\mathbf{p}=\left\{p_{\mu}=N^{-1} N_{\mu}=2 N^{-1} \sum_{i=1}^{n^{o}} C_{\mu, i}^{2}\right\},
$$

with the appropriate normalization condition

$$
\sum_{\mu} p_{\mu}=N^{-1} \sum_{\mu} \gamma_{\mu, \mu}=N^{-1} N=1
$$

The second equality in (6) allows one to define the so called conditional probability matrix $\mathbf{P}$

$$
\mathbf{P}=\left\{P_{\nu \mid \mu}=\left(2 \gamma_{\mu, \mu}\right)^{-1} \gamma_{\mu, \nu}^{2}\right\}
$$

and the following normalization condition

$$
\sum_{\nu} P_{\nu \mid \mu}=\left(2 \gamma_{\mu, \mu}\right)^{-1} \sum_{\nu} \gamma_{\mu, \nu}^{2}=\left(2 \gamma_{\mu, \mu}\right)^{-1}\left(2 \gamma_{\mu, \mu}\right)=1 .
$$

According to OCT, the element of CBO-matrix, $\gamma_{\nu, \mu}$, represents the appropriately renormalized amplitude of the corresponding conditional probability $P_{\nu \mid \mu}$ of "observing" in atomic orbital $\left|\chi_{\nu}\right\rangle$ the electron originally assigned to $\left|\chi_{\mu}\right\rangle$. Consequently, $P_{\nu \mid \mu}>0$ gives rise to direct communication (through-space interaction [14,15]) between AOs, $\mu \rightarrow v$, while a sequence of $r$ serially-connected communications determined by conditional probabilities $P_{v \mid \mu}^{r}$,

$$
\mu \rightarrow \pi^{1} \rightarrow \ldots \rightarrow \pi^{r-1} \rightarrow \nu, \quad P_{\nu \mid \mu}^{r}=P_{\pi^{1} \mid \mu} \cdots P_{\nu \mid \pi^{r-1}}>0
$$

refers to indirect AO-communication (through-bridge interaction [14-16]). It has to be noticed that such information-propagation cascade of AO-channels one can recognize as an analogue to the time-homogeneous Markov chain with a finite state space [13]; then $P_{v \mid \mu}^{r}$ represents the relevant $r$-step transition probability.

Due to idempotency relation (6) the electron distribution $\mathbf{p}$ (row vector) is said to be stationary in the sense of Markov if:

$$
\mathbf{p}=\mathbf{p} \mathbf{P} \quad \text { or else } \quad \mathbf{P}^{T} \mathbf{p}^{T}=1 \mathbf{p}^{T}
$$

As stated by the Perron-Frobenius theorem [17], for any arbitrary matrix with nonnegative elements of each column normalized to 1 there is always at least one stationary probability distribution. In fact, as follows from the second equality of the last equation, the stationary distribution can be ragarded as a normalized left eigenvector of the conditional probability matrix associated with the eigenvalue of 1 . Therefore, for 
given matrix $\mathbf{P}$ one can find the relevant vector $\mathbf{p}$ by solving the eigenproblem (12) but, in general, the resulting probability distribution is not guaranteed to be unique, i.e. there can be more that one eigenvector of $\mathbf{P}$ associated with the eigenvalue 1 . It follows from the Perron-Frobenius theorem that the uniqueness of the stationary electron distribution is always assured if the $x$ th power of the conditional probability matrix converges to a rank-one matrix in which each row is the stationary distribution in question,

$$
\lim _{x \rightarrow \infty} \mathbf{P}^{x}=\mathbf{1} \mathbf{p}
$$

where 1 stands for the column vector with all entries equal 1. Essentially, for a given conditional probability matrix its stationary distribution $\mathbf{p}$ is non-degenerated provided that:

- matrix P represents an aperiodic Markov chain which virtually means that for all atomic orbitals $\left\{\left|\chi_{\mu}\right\rangle\right\}$ the direct self-communication $\mu \rightarrow \mu$ is always possible, $P(\mu \mid \mu)>0$. In practice, aperiodicity can be always attained by appropriate reduction of the AO-space size, i.e. by removing atomic orbitals for which $\gamma_{\mu, \mu}<\epsilon$, where $\epsilon$ represents the fixed threshold value;

- matrix $\mathbf{P}$ represents an irreducible Markov chain. i.e. if all atomic orbitals are directly and/or indirectly communicated with each other. Also this requirement is straightforward to achieve by eliminating (if present) those AOs for which $\gamma_{\mu \mu}>$ $2-\epsilon$ (ussually core orbitals). The subspace od mutually communicated atomic orbitals defines a single communicating class.

Conditional probability (9) and relation (12) are crucial for the electron population analysis. Indeed, each row of matrix $\mathbf{P}$ allows one to decompose effective electron population $N_{\mu}$ into terms related to localized (diagonal) and emphdelocalized (offdiagonal) populations, i.e. $N_{\mu, \mu}$ and $N_{\mu, \nu}$, respectively:

$$
\begin{aligned}
N_{\mu} & =N\left(p_{\mu} P_{\mu \mid \mu}+\sum_{\nu \neq \mu} p_{\mu} P_{\nu \mid \mu}\right)=N_{\mu}\left(P_{\mu \mid \mu}+\sum_{\nu \neq \mu} P_{\nu \mid \mu}\right) \\
& =N_{\mu, \mu}+\sum_{\nu \neq \mu} N_{\mu, \nu} .
\end{aligned}
$$

In atomic resolution the electron population on atom $\mathrm{X}$ can be decomposed as follows:

$$
N_{X}=N_{X, X}+V_{X}=\sum_{\mu \in X} \sum_{\nu \in X} N_{\mu, \nu}+\sum_{\mu \in X} \sum_{\nu \notin X} N_{\mu, \nu} .
$$

Here, $N_{X, X}$ stands for the number of electrons well localized on atom $\mathrm{X}$ (noninteracting "chemically" with electrons from other atoms, e.g. lone pairs, core electrons) and $V_{X}$ is the atomic-covalency index counting up the population of electrons delocalized over chemical bonds with all other atoms, 


$$
V_{X}=\sum_{Y \neq X}\left(\sum_{\mu \in X} \sum_{\nu \in Y} N_{\mu, v}\right)=\sum_{Y \neq X} W_{X, Y} .
$$

$W_{X, Y}$ stands for the standard (quadratic) bond-covalency index originally proposed by Wiberg [18]. However, one should realize that electron populations and covalencies from Eqs. (15)-(16) calculated within representation of orthogonal AOs assume reliable and chemically meaningful values only when minimal set of conventionally atom-assigned atomic orbitals is used (this problem has been recently reexamined within the framework of OCT-approach in [19]). Such minimal basis set of orthogonal atomic orbitals one can always obtain using various methods, e.g. [20,21].

\section{Stationarity without idempotency}

Unfortunately, Definitions (5)-(10) are correct only for one-determinant wavefunctions of closed-shell systems. In typical CI-type calculations the electron density matrix $\Gamma$ of the multi-determinant wavefunction represented by $\left|\Psi_{C I}\right\rangle$ is commonly expressed by means of its eigenvectors - natural orbitals $|\Theta\rangle$, and the corresponding eigenvalues-occupation numbers $\lambda$,

$$
\hat{\Gamma}\left|\Theta_{i}\right\rangle=\lambda_{i}\left|\Theta_{i}\right\rangle, \quad i=1, \ldots, n,
$$

where the electron density operator $\hat{\Gamma}$ is defined as follows:

$$
\hat{\Gamma}=\sum_{i=1}^{n} \lambda_{i} \hat{P}_{i}=\sum_{i=1}^{n} \lambda_{i}\left|\Theta_{i}\right\rangle\left\langle\Theta_{i}\right| .
$$

Then

$$
\Gamma=\langle\chi|\hat{\Gamma}| \chi\rangle=\langle\chi \mid \Theta\rangle \lambda\langle\Theta \mid \chi\rangle=\mathcal{C} \lambda \mathcal{C}^{\dagger}
$$

with normalization $\operatorname{Tr} \boldsymbol{\Gamma}=N$ and the electron probability distribution $\mathbf{p}$ is determined by diagonal elements $\left\{\Gamma_{\mu, \mu}\right\}$

$$
\mathbf{p}=\left\{p_{\mu}=N^{-1} \Gamma_{\mu, \mu}=N^{-1} \sum_{i=1}^{n} \lambda_{i} \mathcal{C}_{\mu, i}^{2}\right\}
$$

However, since the electron density operator $\hat{\Gamma}$ is generally not idempotent,

$$
\hat{\Gamma}^{2}=\sum_{i=1}^{n} \lambda_{i}^{2} \hat{P}_{i}^{2}=\sum_{i=1}^{n} \lambda_{i}^{2} \hat{P}_{i} \neq \sum_{i=1}^{n} \lambda_{i} \hat{P}_{i}=\hat{\Gamma},
$$

normalization condition (10) cannot be fulfilled and hence off-diagonal elements of the relevant density matrix, $\left\{\Gamma_{\mu, \nu}\right\}$ cannot be further regarded as amplitudes 
of corresponding conditional probabilities. In other words, for the hypothetical conditional probability matrix $\mathbf{P}^{\prime}$ obtained from appropriate renormalization of each row of matrix of elements $\left\{\Gamma_{\mu, \nu}^{2}\right\}$ its stationary distribution $\mathbf{p}^{\prime}$ (left eigenvector associated with the eigenvalue 1) differs from $\mathbf{p}$ and

$$
\lim _{x \rightarrow \infty} \mathbf{p} \mathbf{P}^{\prime}=\mathbf{p}^{\prime} \neq \mathbf{p}
$$

To solve this problem we propose in this paper the following alternative definition of conditional probability $P_{v \mid \mu}$, by means of natural orbitals and their occupation numbers:

$$
\mathbf{P}=\left\{P_{\nu \mid \mu}=\Gamma_{\mu, \mu}^{-1} \sum_{i=1}^{n} \lambda_{i} B_{\mu, v}^{i}\right\}
$$

where

$$
B_{\mu, \nu}^{i}=\mathcal{C}_{\mu, i} \mathcal{C}_{v, i}\left(\mathcal{C}_{\mu, i} \mathcal{C}_{\nu, i}+2 \sum_{j=1}^{i-1} \mathcal{C}_{\mu, j} \mathcal{C}_{\nu, j}\right)
$$

Therefore, one can interpret $B_{\mu, \nu}^{i}$ as the contribution of $i$ th NO to the corresponding electron population $N_{\mu, v}$ provided that all orbitals $\left\{\left|\Theta_{j}\right\rangle, j=1, \ldots, i-1\right\}$ are fully occupied.

In the one-determinant case of closed-shell molecular system all $n^{o}$ occupied natural orbitals correspond to degenerated eigenvalue $\lambda_{i}=2$ and therefore can be easily separated from the (usually larger) subspace of $n^{v}$ unoccupied orbitals $\left(\lambda_{i}=0\right)$. Due to this separation and degeneracy of $\left\{\lambda_{i}\right\}$ it can be proved that both definitions of conditional probability $P_{\nu \mid \mu}$, (9) and (23), are fully equivalent, regardless of orbital order:

$$
\begin{aligned}
& P_{\nu \mid \mu}=\Gamma_{\mu, \mu}^{-1} \sum_{i=1}^{n} \lambda_{i} B_{\mu, \nu}^{i}=2 \Gamma_{\mu, \mu}^{-1} \sum_{i=1}^{n^{o}} B_{\mu, \nu}^{i} \\
& =2 \Gamma_{\mu, \mu}^{-1}\left(\sum_{i=1}^{n^{o}} \mathcal{C}_{\mu, i}^{2} \mathcal{C}_{\nu, i}^{2}+2 \sum_{i=1}^{n^{o}} \sum_{j=1}^{i-1} \mathcal{C}_{\mu, i} \mathcal{C}_{\nu, i} \mathcal{C}_{\mu, j} \mathcal{C}_{\nu, j}\right) \\
& =2 \Gamma_{\mu, \mu}^{-1}\left(\sum_{i=1}^{n^{o}} \mathcal{C}_{\mu, i} \mathcal{C}_{\nu, i}\right)\left(\sum_{j=1}^{n^{o}} \mathcal{C}_{\mu, j} \mathcal{C}_{\nu, j}\right) \\
& =2 \Gamma_{\mu, \mu}^{-1}\left(2^{-1} \Gamma_{\mu, \nu}\right)\left(2^{-1} \Gamma_{\mu, \nu}\right)=\left(2 \Gamma_{\mu, \mu}\right)^{-1} \Gamma_{\mu, \nu}^{2}
\end{aligned}
$$

For one-determinant wavefuntions the atomic charges and Wiberg-type bond covalencies (14)-(16) based on the density matrix (5) are commonly known to be invariant 
with respect to unitary transformation of molecular orbitals. Thus, from the equivalence of definitions (9) and (23) one can deduce the same transformational properties of conditional probabilities (23).

It is also straightforward to prove that, for the newly proposed definition (23), stationarity of probability distribution $\mathbf{p}$ is preserved for any arbitrary permutation of eigenvectors and eigenvalues of density matrix. First, let us sum up elements $B_{\mu, v}^{i}$ over all atomic orbitals,

$$
\begin{aligned}
& \sum_{\nu} B_{\mu, \nu}^{i}=\sum_{v} \mathcal{C}_{\mu, i}^{2} \mathcal{C}_{\nu, i}^{2}+2 \sum_{\nu} \sum_{j=1}^{i-1} \mathcal{C}_{\mu, i} \mathcal{C}_{v, i} \mathcal{C}_{\mu, j} \mathcal{C}_{v, j} \\
& =\mathcal{C}_{\mu, i}^{2}\left(\sum_{\nu} \mathcal{C}_{v, i}^{2}\right)+2 \sum_{j=1}^{i-1} \mathcal{C}_{\mu, i} \mathcal{C}_{\mu, j}\left(\sum_{\nu} \mathcal{C}_{\nu, i} \mathcal{C}_{v, j}\right) \\
& =\mathcal{C}_{\mu, i}^{2}+2 \sum_{j=1}^{i-1} \mathcal{C}_{\mu, i} \mathcal{C}_{\mu, j} \delta_{i, j}=\mathcal{C}_{\mu, i}^{2}
\end{aligned}
$$

The last equality in the preceding equation results from orthonormality of natural orbitals, $\left\langle\Theta_{i} \mid \Theta_{j}\right\rangle=\delta_{i, j}$. Now, using the result from (26) we can easily prove the appropriate normalization requirement,

$$
\begin{aligned}
& \sum_{\nu} P_{\nu \mid \mu}=\Gamma_{\mu, \mu}^{-1} \sum_{i=1}^{n} \lambda_{i}\left(\sum_{\nu} B_{\mu, \nu}^{i}\right)= \\
& \Gamma_{\mu, \mu}^{-1} \sum_{i=1}^{n} \lambda_{i} \mathcal{C}_{\mu, i}^{2}=\Gamma_{\mu, \mu}^{-1} \Gamma_{\mu, \mu}=1 .
\end{aligned}
$$

Since electron probabilities (and the corresponding populations) are invariant with respect to interchange of any pair of natural orbitals there are formally $r_{P}=n$ ! different matrices $\mathbf{P}$ satisfying (12) for non-degenerated $\lambda$ (i.e. $g_{s}=1$ for $s=1, \ldots, n$ ). However, if degeneracy occurs (or certain eigenvalues below a fixed threshold can be neglected) and there are only $n^{\prime}$ different eigenvalues (i.e. $g_{s} \geq 1$ for $s=1, \ldots, n^{\prime}$ ) then the number of possible conditional probability matrices reduces to

$$
r_{P}=\left(\prod_{s}^{n^{\prime}} g_{s} !\right)^{-1} n !
$$

where $g_{s}$ stands for the degeneracy of $s$ th possible eigenvalue.

In the case of one-determinant calculations subspaces of occupied and virtual orbitals are strictly separated and there are exactly $n^{\circ}$ ! permutations of $\lambda$ that lead to the same conditional probability matrix. Furthermore, due to orthonormality of natural orbitals and basis functions, the maximal separation of these subspaces ensures that all $P_{v \mid \mu} \geq 0$. All permutations of "mixed" occupied and unoccupied NOs can 
lead to negative values of $P_{\nu \mid \mu}$; this is mainly because of predominatingly anti-bonding character of virtual subspace with relation to the occupied one. This facts allow one to draw a conclusion that for the multi-determinant calculations the natural generalization of the maximal separation requirement is the following condition:

$$
\sum_{i=1}^{n-1}\left(\lambda_{i}-\lambda_{i+1}\right)^{2}=\text { minimum }
$$

Thus, for ground-state systems the conditional probability matrix $\mathbf{P}$ obtained from permutations of NOs for which $\lambda_{1} \leq \ldots \leq \lambda_{i} \leq \lambda_{i+1} \leq \ldots \leq \lambda_{n}$, reveals the minimal dependence on order of NOs within the particular (conventionally separated) subspace, high occupied $\left(\lambda_{i} \approx 2\right)$ or low occupied orbitals $\left(\lambda_{i} \approx 0\right)$.

\section{Summary}

It was the main goal of this short paper to demonstrate that idempotency property of the density operator is not an indispensable condition for the stationarity of electron probability distribution in molecular systems. We have introduced a simple method of evaluation of conditional probabilities for the multi-determinant wavefunction. Moreover, the newly proposed definition based on natural orbitals successfully displaces hitherto prevailing one for the one-determinant wavefunctions. Since presented methodology introduces some arbitrariness due to orbital order, it has been argued to use the criterion of maximal separation of nearly-degenerated orbital subspaces in ground-state calculations.

The proposed method can be also extended to cover open-shell molecular systems as well as the excited states. However, this requires a more insightful investigation and will be the subject of the seperate article.

Open Access This article is distributed under the terms of the Creative Commons Attribution License which permits any use, distribution, and reproduction in any medium, provided the original author(s) and the source are credited.

\section{References}

1. R.S. Mulliken, J. Chem. Phys. 23, 1833 (1955)

2. R.S. Mulliken, J. Chem. Phys. 23, 1841 (1955)

3. P.O. Löwdin, J. Chem. Phys. 18, 365 (1950)

4. D. Szczepanik, J. Mrozek, Chem. Phys. Lett. 521, 157 (2012)

5. A.E. Reed, R.B. Weinstock, F. Weinhold, J. Chem. Phys. 83, 735 (1985)

6. R.F.W. Bader, P.M. Beddall, P.E. Cade, J. Am. Chem. Soc. 93, 3095 (1971)

7. F.L. Hirshfeld, Theor. Chim. Acta. 44, 129 (1977)

8. U.C. Singh, P.A. Koolman, J. Comput. Chem. 5, 129 (1984)

9. F. Jensen, Introduction to Computational Chemistry, 2nd edn. (Wiley, West Sussex, 2007)

10. R.F. Nalewajski, Information Theory of Molecular Systems (Elsevier, Amsterdam, 2006)

11. R.F. Nalewajski, Information Origins of The Chemical Bond (Nova Science Publishers, Hauppauge, 2010)

12. R.F. Nalewajski, D. Szczepanik, J. Mrozek, Adv. Quantum Chem. 61, 1 (2011) 
13. A.A. Markov, Sci. Context 19(4), 591 (2006)

14. R.F. Nalewajski, J. Math. Chem. 49, 371 (2011)

15. R.F. Nalewajski, Int. J. Quantum Chem. 112, 2355 (2012)

16. R.F. Nalewajski, J. Math. Chem. 49, 546 (2011)

17. J. Keener, SIAM Rev. 35, 80 (1993)

18. K.B. Wiberg, Tetrahedron 24, 1083-1096 (1968)

19. R.F. Nalewajski, D. Szczepanik, J. Mrozek, J. Math. Chem. 50, 1437 (2012)

20. D. Szczepanik, J. Mrozek, Comput. Theor. Chem. 996, 103 (2012)

21. W.C. Lu, C.Z. Wang, M.W. Schmidt, L. Bytautas, K.M. Ho, K. Ruedenberg, J. Chem. Phys. 120, 2629 (2004) 